\title{
Ferroelectric atomic displacement in multiferroic tetragonal perovskite $\mathrm{Sr}_{1 / 2} \mathrm{Ba}_{1 / 2} \mathrm{MnO}_{3}$
}

\author{
D. Okuyama, ${ }^{1, *}$ K. Yamauchi $\odot,{ }^{2}$ H. Sakai $\odot,{ }^{3,4}$ Y. Taguchi, ${ }^{5}$ Y. Tokura,${ }^{5,6}$ K. Sugimoto, ${ }^{7}$ T. J. Sato, ${ }^{1}$ and T. Oguchi $\odot^{2}$ \\ ${ }^{1}$ Institute of Multidisciplinary Research for Advanced Materials, Tohoku University, Katahira 2-1-1, Sendai 980-8577, Japan \\ ${ }^{2}$ ISIR-SANKEN, Osaka University, 8-1 Mihogaoka, Ibaraki, Osaka 567-0047, Japan \\ ${ }^{3}$ Department of Physics, Osaka University, Toyonaka, Osaka 560-0043, Japan \\ ${ }^{4}$ PRESTO, Japan Science and Technology Agency, Kawaguchi, Saitama 332-0012, Japan \\ ${ }^{5}$ RIKEN Center for Emergent Matter Science, Wako 351-0198, Japan \\ ${ }^{6}$ Tokyo College and Department of Applied Physics, University of Tokyo, Tokyo 113-8656, Japan \\ ${ }^{7}$ JASRI SPring-8, Hyogo 679-5198, Japan
}

(Received 5 February 2020; revised 14 May 2020; accepted 27 May 2020; published 8 July 2020)

\begin{abstract}
We investigate the crystal structure in multiferroic tetragonal perovskite $\mathrm{Sr}_{1 / 2} \mathrm{Ba}_{1 / 2} \mathrm{MnO}_{3}$ with high accuracy of the order of $10^{-3} \AA$ for an atomic displacement. A large atomic displacement of the $\mathrm{Mn}$ ion from the centrosymmetric position, comparable with the off-centering distortion in the tetragonal ferroelectric $\mathrm{BaTiO}_{3}$, is observed in the ferroelectric phase $\left(T_{\mathrm{N}} \leqslant T \leqslant T_{\mathrm{C}}\right)$. In stark contrast, in the multiferroic phase $\left(T \leqslant T_{\mathrm{N}}\right)$, the atomic displacement for the $\mathrm{Mn}$ ion is suppressed, but those for $\mathrm{O}$ ions are enlarged. The atomic displacements in the polar crystal structures are also analyzed in terms of the ferroelectric modes. In the ferroelectric phase, the atomic displacements are decomposed into dominant positive Slater, negative Last, and small positive Axe modes. The suppression of Slater and Last modes, the sign change of the Last mode, and the enlargement of the Axe mode are found in the multiferroic phase. The ferroelectric distortion is well reproduced by a first-principles calculation based on the Berry phase method, providing additional information on competing mechanisms to induce the ferroelectric polarization, electronic $p$ - $d$ hybridization versus magnetic exchange striction. The quantitative comparison between the experimental result and the theoretical calculation leads to a better understanding of the ferroelectric polarization in the multiferroic phase.
\end{abstract}

DOI: 10.1103/PhysRevResearch.2.033038

\section{INTRODUCTION}

Numerous investigations of ferroelectricity to elucidate the driving mechanism and to develop device applications have been presented for ferroelectric perovskite oxides $\left(A B \mathrm{O}_{3}\right)$ [1-6]. In the archetypal ferroelectric $\mathrm{BaTiO}_{3}$, the electronic $p$ - $d$ hybridization (covalency) between empty $d$ orbitals of a transition metal and filled $2 p$ orbitals of oxygen causes a large ferroelectric polarization $[1,7]$. In contrast, as ferroelectricity of an unconventional origin, magnetic-ordering-induced ferroelectric (so-called multiferroic) materials have also been extensively investigated since a large nonlinear magnetoelectric effect was found in the perovskite $\mathrm{TbMnO}_{3}$ [8-11]. It is well known that the ferroelectric polarization of many multiferroic materials is far smaller than that of the archetypal ferroelectric $\mathrm{BaTiO}_{3}$. Nevertheless, rather large ferroelectric polarizations among the multiferroic materials have been observed in the perovskite $\mathrm{BiFeO}_{3}$ [12-14] and have theoretically been shown in the tetragonal perovskite $\mathrm{BaMnO}_{3}$ [15-17]. In $\mathrm{BaMnO}_{3}$,

\footnotetext{
*okudaisu@ tohoku.ac.jp

Published by the American Physical Society under the terms of the Creative Commons Attribution 4.0 International license. Further distribution of this work must maintain attribution to the author(s) and the published article's title, journal citation, and DOI.
}

it is proposed that a large ferroelectricity is induced by the off-centering distortion of the $\mathrm{Mn}$ and $\mathrm{O}$ ions originating from the $p$ - $d$ hybridization between filled $3 d$ orbitals of manganese and $2 p$ orbitals of oxygen in the paramagnetic phase. Since the magnetic $\mathrm{Mn}^{4+}$ ion directly contributes to the emergence of the ferroelectricity, a large magnetoelectric effect is expected. When ferroelectricities originating from a $p-d$ hybridization and a magnetic interaction coexist, how they compete or concert is still an open question, and $\mathrm{BaMnO}_{3}$ is one of the promising candidates to solve such a question. However, in reality, it is known that the hexagonal structural phase is stable in $\mathrm{BaMnO}_{3}$. Sakai et al. found that $\mathrm{Sr}_{1 / 2} \mathrm{Ba}_{1 / 2} \mathrm{MnO}_{3}$ with a smaller tolerance factor is crystallized in the tetragonal perovskite structure and shows ferroelectricity [18]. Below the magnetic phase transition temperature, the change in the crystal lattice with a large reduction in the ferroelectric polarization is observed in the tetragonal perovskite $\mathrm{Sr}_{1 / 2} \mathrm{Ba}_{1 / 2} \mathrm{MnO}_{3}$ [18]. The mechanism of the ferroelectric polarization upon the magnetic ordering in $\mathrm{Sr}_{1 / 2} \mathrm{Ba}_{1 / 2} \mathrm{MnO}_{3}$ should be quantitatively unveiled by dividing the ferroelectric polarization into the respective contributions from the $p-d$ hybridization and that from the magnetic interaction.

Perovskite $\mathrm{Sr}_{1 / 2} \mathrm{Ba}_{1 / 2} \mathrm{MnO}_{3}$ exhibits two phase transitions, ferroelectric and antiferromagnetic, at $T_{\mathrm{C}} \sim 400 \mathrm{~K}$ and $T_{\mathrm{N}} \sim 185 \mathrm{~K}$ [18], respectively. Here, we call the phases for $T_{\mathrm{N}} \leqslant T \leqslant T_{\mathrm{C}}$ ferroelectric and for $T \leqslant T_{\mathrm{N}}$ multiferroic. Below $T_{\mathrm{C}}$, it is reported that the crystal system changes from 

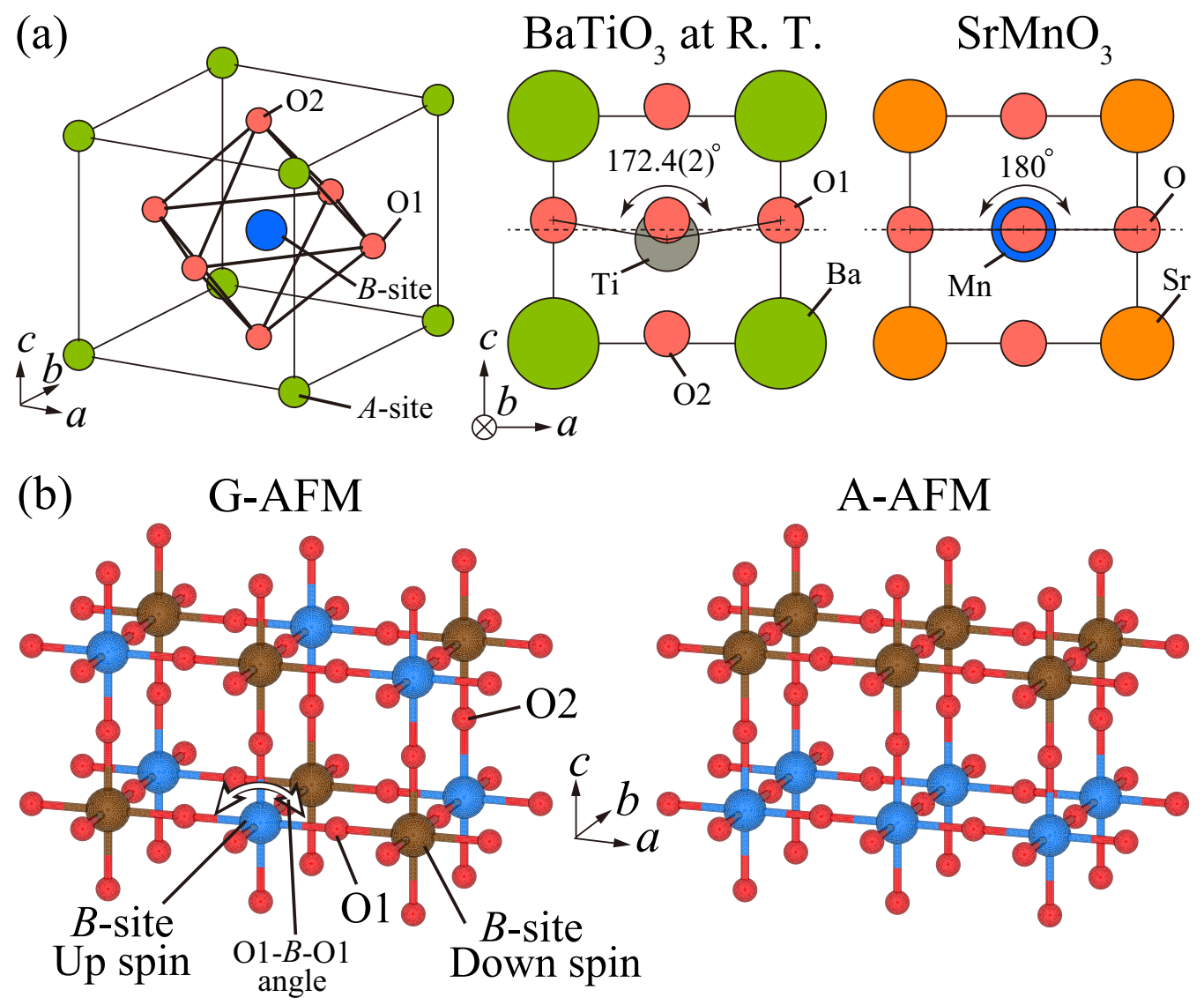

Up spin $\begin{gathered}\mathrm{O} 1-B-\mathrm{O} 1 \\ \text { angle }\end{gathered}$

FIG. 1. (a) Schematic illustrations of the perovskite $A B \mathrm{O}_{3}$ structure. The O1-B-O1 bond angles of $\mathrm{BaTiO}_{3}$ and $\mathrm{SrMnO}_{3}$ are also shown. (b) Schematics to explain the $G$-type antiferromagnetic $\left(G\right.$-AFM) and $A$-type antiferromagnetic $\left(A\right.$-AFM) structures on $A B \mathrm{O}_{3}$, drawn by VESTA [22]. Here, the $A$-site ions are not shown. Blue and brown circles stand for the up and down spins on the $B$ site, respectively. O1 and $\mathrm{O} 2$ sites are indicated by the red circles. In $G$-AFM, the neighbor magnetic moments are aligned antiparallel. In $A$-AFM, the magnetic moments are aligned parallel in the $a-b$ plane with antiparallel coupling with neighbor planes.

centrosymmetric cubic to polar tetragonal, determined from the temperature variation of the $c / a$-lattice-constant ratio. The $c / a$ ratio increases with decreasing temperature below $T_{\mathrm{C}}$ and saturates near $250 \mathrm{~K}$. In contrast, below $T_{\mathrm{N}}$, the $c / a$ ratio turns to decrease with decreasing temperature and saturates below $150 \mathrm{~K}$. From the reduction of $c / a$, it was speculated that the ferroelectric polarization is also reduced. A crystal-structure analysis using the twin-free single crystal was also performed in the ferroelectric phase [18]. The result indicates that the origin of the ferroelectricity is the off-centering distortion of the O1-Mn-O1 bond angle, the same as the tetragonal ferroelectric $\mathrm{BaTiO}_{3}$ [see Fig. 1(a)] [19-21]. In the multiferroic phase, the distortion of O1-Mn-O1 at $50 \mathrm{~K}$ is smaller than that at $225 \mathrm{~K}$ in the ferroelectric phase. $\mathrm{In}\left(\mathrm{Sr}_{1-x} \mathrm{Ba}_{x}\right) \mathrm{MnO}_{3}(0$ $\leqslant x \leqslant 1 / 2$ ), only one magnetically ordered phase, where the antiferromagnetic-transition temperature gradually decreases with increasing $x$, was observed from the temperature dependences of magnetization [18]. In view of the phase continuity from the antiferromagnetic ordered phase of $\mathrm{SrMnO}_{3}[23,24]$, the antiferromagnetic structure in the multiferroic phase is inferred to be $G$ type ( $G$-AFM), in which nearest-neighbor magnetic moments are aligned antiparallel, as shown in Fig. 1(b). The additional proof of the $G$-AFM ground state will be discussed in Sec. III B and in Appendix A. In the earlier study, it was speculated that the ferroelectric polarization can be suppressed to obtain the gain of the magnetic exchange energy [18,25]. To the best of our knowledge, however, a quantitative comparison between the experimental result and the theoretical calculation for the ferroelectric polarization in the multiferroic phase has not yet been done. To quantitatively discuss the suppression mechanism of the ferroelectric polarization, information about the atomic displacements and the frozen ferroelectric modes in the ferroelectric and multiferroic phases is necessary. Nonetheless, in the earlier crystal-structure analysis, the obtained atomic displacements of the ions in multiferroic phase is smaller than the experimental uncertainties [18]. Thus, an accurate crystal-structure analysis in the multiferroic phase of the tetragonal perovskite $\mathrm{Sr}_{1 / 2} \mathrm{Ba}_{1 / 2} \mathrm{MnO}_{3}$ is desired.

In a multiferroic system, an essential contribution of the quantum Berry phase of valence electrons can be revealed by the combined study of accurate structural analysis and first-principles calculation [26]. For tetragonal perovskite $\mathrm{Sr}_{1 / 2} \mathrm{Ba}_{1 / 2} \mathrm{MnO}_{3}$, Giovannetti et al. performed the first-principles density-functional theory (DFT) band simulation and claimed that the ferroelectric polarization caused by $\mathrm{Mn}-\mathrm{O} 2$ hybridization is suppressed by $\mathrm{Mn}-\mathrm{O} 1-\mathrm{Mn}$ superexchange interaction in the $G$-AFM ordering [25]. In their 
study, the crystal structure in the multiferroics phase was theoretically optimized with the generalized gradient approximation (GGA) potential, whereas the comparison with the experimental structure was missing. It is also noteworthy here that a simulation study can provide an ideal magnetic structure that enhances ferroelectricity $[27,28]$. In multiferroic materials, the change in the magnetic structure may induce much larger ferroelectric polarization $[29,30]$. Thus, for the further understanding of the multiferroic properties in tetragonal perovskite $\mathrm{Sr}_{1 / 2} \mathrm{Ba}_{1 / 2} \mathrm{MnO}_{3}$, it is important as well to evaluate the ferroelectric polarizations in various magnetic configurations.

In this paper, we report atomic displacements in the ferroelectric and multiferroic phases of the tetragonal perovskite $\mathrm{Sr}_{1 / 2} \mathrm{Ba}_{1 / 2} \mathrm{MnO}_{3}$ determined by the crystal-structure analyses using the twin-free single crystal and higher- $Q$ diffraction data than in earlier work [18]. With ferroelectric mode analyses, the polar crystal structures in the ferroelectric and multiferroic phases for $\mathrm{Sr}_{1 / 2} \mathrm{Ba}_{1 / 2} \mathrm{MnO}_{3}$ and other tetragonal perovskites were classified. Using a first-principles calculation based on accurate crystal-structure parameters, we quantitatively elucidate the suppression mechanism of the ferroelectric polarization in the multiferroic phase and discuss the possible magnetic structure that enhances the ferroelectric polarization.

\section{EXPERIMENTAL AND COMPUTATIONAL PROCEDURES}

A single crystal of tetragonal perovskite $\mathrm{Sr}_{1 / 2} \mathrm{Ba}_{1 / 2} \mathrm{MnO}_{3}$ was synthesized by a high-pressure treatment on the precursor sample of oxygen-deficient single crystals [18]. A synchrotron $\mathrm{x}$-ray diffraction experiment was performed on BL02B1 at SPring-8, Japan [31]. The photon energy of the incident $x$ rays was tuned to $35.04 \mathrm{keV}$. Using the high-energy $\mathrm{x}$ ray, we can access diffraction peaks with high spatial resolution up to $Q \sim 30 \AA^{-1}$. The single crystal was crushed into cubes with a typical dimension of about $20 \mu \mathrm{m}$. The absorption coefficient $\mu$ is calculated to be $37.85 \mathrm{~cm}^{-1}$. The empirical absorption correction was carried out [32]. The RAPID-AUTO program (Rigaku Corp.) was used to obtain an $F$ table. The CRYSTAL

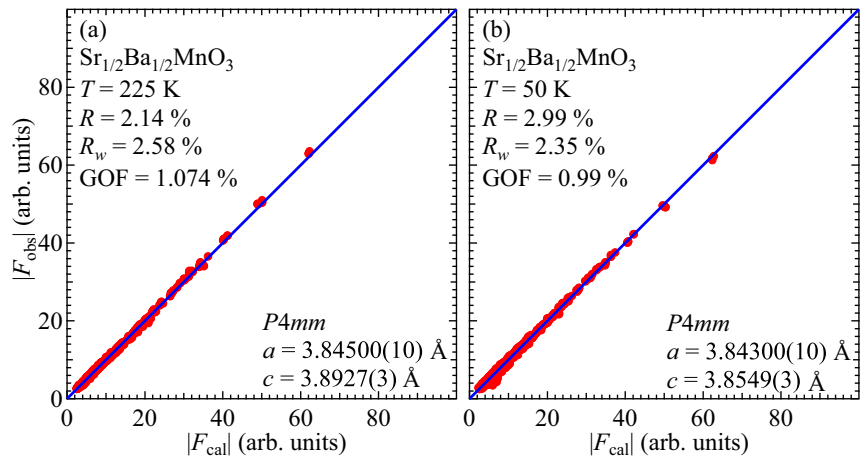

FIG. 2. Comparison between observed $\left(\left|F_{\text {obs }}\right|\right)$ and calculated $\left(\left|F_{\text {cal }}\right|\right)$ structure factors (a) at $225 \mathrm{~K}$ in the ferroelectric phase and (b) at $50 \mathrm{~K}$ in the multiferroic phase of tetragonal perovskite $\mathrm{Sr}_{1 / 2} \mathrm{Ba}_{1 / 2} \mathrm{MnO}_{3}$.

STRUCTURE (Rigaku Corp.) program was used to analyze the crystal structure from the $F$ table. In the crystal-structure analysis in the multiferroic phase at $50 \mathrm{~K}$, the isotropic atomic displacement parameter $B_{\text {iso }}$ was used for the $\mathrm{Ba} / \mathrm{Sr}$ site.

First-principles calculations were performed using the VASP code [33] within the GGA $+U$ [34] formalism with various $U$ values for $\mathrm{Mn} 3 d$ states. In addition, we employed the Heyd-Scuseria-Ernzerhof (HSE06) screened hybrid functional method [35], which mixes the exact nonlocal Fock exchange and the density-functional parametrized exchange. HSE06 is known to improve the evaluation of the band gap energy and the structural distortion, with respect to GGA + $U$ approaches [36]. The cutoff energy for the plane-wave expansion of the wave functions was set to $400 \mathrm{eV}$, and a $\boldsymbol{k}$-point shell of $(4,4,3)$ was used for the Brillouin zone integration according to the Monkhorst-Pack special point mesh. The convergence of ferroelectric polarization with respect to cutoff energy and $\boldsymbol{k}$-point sampling is carefully checked in Appendix B. The crystal structure was optimized with respect to internal atomic coordinates until the remaining forces were less than $1 \mathrm{meV} / \AA$ while the lattice parameters were kept at the experimental values.

TABLE I. Structure parameters of $\mathrm{Sr}_{1 / 2} \mathrm{Ba}_{1 / 2} \mathrm{MnO}_{3}$ at $225 \mathrm{~K}$ in the ferroelectric phase (space group P4mm, No. 99). Some 22 953 reflections were observed, and 3455 of them are independent. Seventeen variables were used for the refinement. The lattice parameters are $a$ $=3.84500(10) \AA$ and $c=3.8927(3) \AA$. The reliability factors are $R=2.14 \%, R_{w}=2.58 \%$, and goodness of fit $(\mathrm{GOF})=1.074$. In the tables, $x, y$, and $z$ are the fractional coordinates. Anisotropic atomic displacement parameters are represented as $U_{11}, U_{22}, U_{33}, U_{12}, U_{13}$, and $U_{23}$.

\begin{tabular}{|c|c|c|c|c|c|c|}
\hline & Site & $x$ & $y$ & $z$ & $B_{\mathrm{eq}}$ & Occupancy \\
\hline $\mathrm{Ba} / \mathrm{Sr}$ & $1 a$ & 0 & 0 & 0 & $0.36(5)$ & $1 / 2$ \\
\hline $\mathrm{Mn}$ & $1 b$ & $1 / 2$ & $1 / 2$ & $0.48173(13)$ & $0.3032(15)$ & 1 \\
\hline $\mathrm{O} 1$ & $2 c$ & 0 & $1 / 2$ & $0.5056(4)$ & $0.609(7)$ & 1 \\
\hline \multirow[t]{2}{*}{$\mathrm{O} 2$} & $1 b$ & $1 / 2$ & $1 / 2$ & $0.0090(5)$ & $0.658(8)$ & 1 \\
\hline & $U_{11}\left(\AA^{2}\right)$ & $U_{22}\left(\AA^{2}\right)$ & $U_{33}\left(\AA^{2}\right)$ & $U_{12}\left(\AA^{2}\right)$ & $U_{13}\left(\AA^{2}\right)$ & $U_{23}\left(\AA^{2}\right)$ \\
\hline $\mathrm{Ba}$ & $0.00529(7)$ & $0.00529(7)$ & $0.00313(11)$ & 0 & 0 & 0 \\
\hline $\mathrm{Sr}$ & $0.00409(9)$ & $0.00409(9)$ & $0.00009(8)$ & 0 & 0 & 0 \\
\hline Mn & $0.00450(3)$ & $0.00450(3)$ & $0.00252(6)$ & 0 & 0 & 0 \\
\hline $\mathrm{O} 1$ & $0.00465(13)$ & $0.00908(19)$ & $0.0094(2)$ & 0 & 0 & 0 \\
\hline $\mathrm{O} 2$ & $0.00935(19)$ & $0.00935(19)$ & $0.0063(2)$ & 0 & 0 & 0 \\
\hline
\end{tabular}


TABLE II. Structure parameters of $\mathrm{Sr}_{1 / 2} \mathrm{Ba}_{1 / 2} \mathrm{MnO}_{3}$ at $50 \mathrm{~K}$ in the multiferroic phase (space group P4mm, No. 99). Some 23 321 reflections were observed, and 3409 of them are independent. Fourteen variables were used for the refinement. The lattice parameters are $a=3.84300(10) \AA$ and $c=3.8549(3) \AA$. The reliability factors are $R=2.99 \%, R_{w}=2.35 \%$, and GOF $=0.99$. In the $\mathrm{Ba} / \mathrm{Sr}$ site, the isotropic atomic displacement parameter is used for the crystal-structure analysis.

\begin{tabular}{|c|c|c|c|c|c|c|}
\hline & Site & $x$ & $y$ & $z$ & $B_{\text {iso }} / B_{\text {eq }}$ & Occupancy \\
\hline $\mathrm{Ba} / \mathrm{Sr}$ & $1 a$ & 0 & 0 & 0 & $0.31(5)$ & $1 / 2$ \\
\hline $\mathrm{Mn}$ & $1 b$ & $1 / 2$ & $1 / 2$ & $0.4954(4)$ & $0.298(2)$ & 1 \\
\hline $\mathrm{O} 1$ & $2 c$ & 0 & $1 / 2$ & $0.5105(13)$ & $0.500(8)$ & 1 \\
\hline \multirow[t]{2}{*}{$\mathrm{O} 2$} & $1 b$ & $1 / 2$ & $1 / 2$ & $0.0161(11)$ & $0.472(12)$ & 1 \\
\hline & $U_{11}\left(\AA^{2}\right)$ & $U_{22}\left(\AA^{2}\right)$ & $U_{33}\left(\AA^{2}\right)$ & $U_{12}\left(\AA^{2}\right)$ & $U_{13}\left(\AA^{2}\right)$ & $U_{23}\left(\AA^{2}\right)$ \\
\hline $\mathrm{Mn}$ & $0.00355(3)$ & $0.00355(3)$ & $0.00424(15)$ & 0 & 0 & 0 \\
\hline $\mathrm{O} 1$ & $0.00414(16)$ & $0.0068(2)$ & $0.0080(3)$ & 0 & 0 & 0 \\
\hline $\mathrm{O} 2$ & $0.00680(19)$ & $0.00680(19)$ & $0.0043(5)$ & 0 & 0 & 0 \\
\hline
\end{tabular}

\section{RESULTS AND DISCUSSION}

\section{A. Synchrotron $x$-ray diffraction and crystal-structure analysis}

The synchrotron x-ray diffraction experiments were carried out in the ferroelectric $(T=225 \mathrm{~K})$ and multiferroic $(T=$ $50 \mathrm{~K}$ ) phases of the tetragonal perovskite $\mathrm{Sr}_{1 / 2} \mathrm{Ba}_{1 / 2} \mathrm{MnO}_{3}$. All observed diffraction spots can be indexed by those of the $P 4 m m$ space group. By using these data sets, we performed crystal-structure analyses. Here, the $A$-site ion is fixed at the centrosymmetric position. Comparisons between observed and calculated structure factors are shown in Fig. 2. The structural parameters at 225 and $50 \mathrm{~K}$ are summarized in Tables I and II, respectively. Schematic views of the atomic displacements in the ferroelectric and multiferroic phases of the tetragonal perovskite $\mathrm{Sr}_{1 / 2} \mathrm{Ba}_{1 / 2} \mathrm{MnO}_{3}$ are shown in Figs. 3(a) and 3(b). As a reference, the O1-Mn-O1 bond angles of tetragonal $\mathrm{BaTiO}_{3}$ and cubic $\mathrm{SrMnO}_{3}$ are also shown in Fig. 1(a). In the ferroelectric phase of the tetragonal perovskite $\mathrm{Sr}_{1 / 2} \mathrm{Ba}_{1 / 2} \mathrm{MnO}_{3}$, the atomic displacements along the $c$ axis at $225 \mathrm{~K}$ are $0.0711(5) \AA$ for the $\mathrm{Mn}$ ion, $0.0218(16) \AA$ for O1 ( $2 c$ site), and $0.0350(19) \AA$ for O2 (1b site), which are of the same order of magnitude as those reported by the earlier study [18]. The off-centering distortion can be estimated by the O1-Mn-O1 bond angle as $174.45(13)^{\circ}$ at $225 \mathrm{~K}$ in the ferroelectric phase, comparable with that of tetragonal $\mathrm{BaTiO}_{3}$ [37].

The atomic displacements and O1-Mn-O1 distortion at $50 \mathrm{~K}$ in the multiferroic phase are respectively changed to $0.0177(15) \AA$ for the $\mathrm{Mn}$ ion, 0.040(5) $\AA$ for O1, and $0.062(4) \AA$ for $\mathrm{O} 2$ and $176.5(2)^{\circ}$. In this study, since the atomic displacements are determined with the accuracy of the $10^{-3} \AA$ order, we observe that the atomic displacements of $\mathrm{O}$ ions are larger than that of the $\mathrm{Mn}$ ion in the multiferroic phase. This enlarged atomic displacement of the $\mathrm{O}$ ions cannot be explained only by the suppression of the off-centering distortion. The reason for this enlargement will be discussed later.

The observed atomic displacements and O1-Mn-O1 bond angles in Figs. 3(a) and 3(b) are summarized and compared with those for other tetragonal perovskites $A B \mathrm{O}_{3}$ in Table III. The relatively large and negative atomic displacement of the $B$ site at $225 \mathrm{~K}$ in the ferroelectric phase of $\mathrm{Sr}_{1 / 2} \mathrm{Ba}_{1 / 2} \mathrm{MnO}_{3}$ is comparable with those of tetragonal $\mathrm{BaTiO}_{3}$ and $\mathrm{KNbO}_{3}$. In stark contrast, the atomic displacement of the $B$ site at $50 \mathrm{~K}$ in the multiferroic phase of $\mathrm{Sr}_{1 / 2} \mathrm{Ba}_{1 / 2} \mathrm{MnO}_{3}$ is much smaller than those of oxygen ions, despite still being negative, which is incompatible with other tetragonal perovskite ferroelectrics. However, by comparing only the atomic displacement of each ion, it is difficult to discuss the origins of the ferroelectric polarizations. To discuss them for the multiferroic phase, a proper basis enabling us to compare all the ferroelectric atomic displacements with those of other tetragonal perovskite ferroelectrics is necessary.

Next, we analyzed the observed atomic displacements by the ferroelectric modes to compare the ferroelectric and multiferroic phases of the tetragonal perovskite $\mathrm{Sr}_{1 / 2} \mathrm{Ba}_{1 / 2} \mathrm{MnO}_{3}$

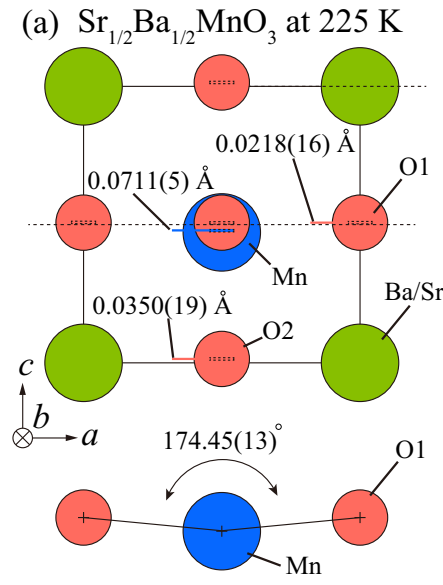

(c) Slater mode

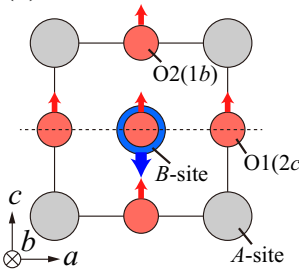

Last mode

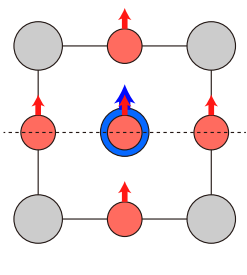

(b) $\mathrm{Sr}_{1 / 2} \mathrm{Ba}_{1 / 2} \mathrm{MnO}_{3}$ at $50 \mathrm{~K}$

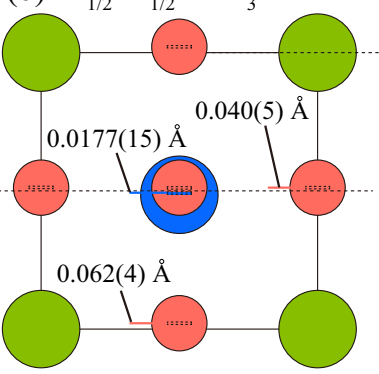

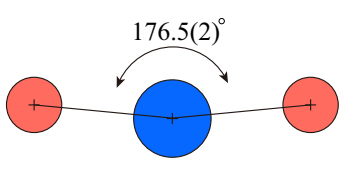

Axe mode

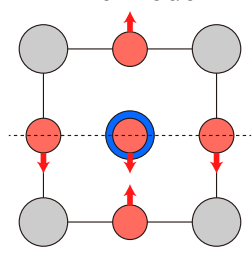

FIG. 3. (a) and (b) The atomic displacements and O1-Mn-O1 bond angles of tetragonal perovskite $\mathrm{Sr}_{1 / 2} \mathrm{Ba}_{1 / 2} \mathrm{MnO}_{3}$ at (a) $225 \mathrm{~K}$ in the ferroelectric phase and (b) $50 \mathrm{~K}$ in the multiferroic phase. (c) Atomic displacements of the respective ferroelectric modes on the perovskite $\mathrm{ABO}_{3}$. Red and blue arrows stand for the directions of the displacement for the $\mathrm{O}$ and $B$-site ions, respectively. 
TABLE III. Frozen ferroelectric modes estimated from the atomic displacements for $B$-site and $\mathrm{O}$ ions in the tetragonal ferroelectric and multiferroic phases. $S_{\text {Slater }}, S_{\text {Last }}$, and $S_{\text {Axe }}$ stand for the coefficients of Slater, Last, and Axe ferroelectric modes, respectively. The contribution ratios from $\left|S_{\text {Slater }}\right|,\left|S_{\text {Last }}\right|$, and $\left|S_{\mathrm{Axe}}\right|$ are also shown in brackets. $\xi_{B}, \xi_{\mathrm{O} 1}$, and $\xi_{\mathrm{O} 2}$ are, respectively, the atomic displacements for $B, \mathrm{O} 1$, and $\mathrm{O} 2$ sites. Here, we selected the sign of the atomic displacement $\xi_{B}$ so that $S_{\text {Slater }}$ is positive. $\angle_{\mathrm{O} 1 B \mathrm{O} 1}$ stands for the distortion of the O1-B-O1 bond angle.

\begin{tabular}{|c|c|c|c|c|c|c|c|}
\hline & $\xi_{B}(\AA)$ & $\xi_{\mathrm{O} 1}(\AA)$ & $\xi_{\mathrm{O} 2}(\AA)$ & $S_{\text {Slater }}$ & $S_{\text {Last }}$ & $S_{\text {Axe }}$ & $\angle_{\mathrm{O} 1 B \mathrm{O} 1}(\mathrm{deg})$ \\
\hline $\begin{array}{l}\mathrm{Sr}_{1 / 2} \mathrm{Ba}_{1 / 2} \mathrm{MnO}_{3} \\
\quad(225 \mathrm{~K})\end{array}$ & $-0.0711(5)$ & $0.0218(16)$ & $0.0350(19)$ & $\begin{array}{c}0.0519(13) \\
{[70 \%]}\end{array}$ & $\begin{array}{c}-0.0134(9) \\
{[18 \%]}\end{array}$ & $\begin{array}{c}0.009(2) \\
{[12 \%]}\end{array}$ & $174.45(13)$ \\
\hline $\begin{array}{l}\mathrm{Sr}_{1 / 2} \mathrm{Ba}_{1 / 2} \mathrm{MnO}_{3} \\
\quad(50 \mathrm{~K})\end{array}$ & $-0.0177(15)$ & $0.040(5)$ & $0.062(4)$ & $\begin{array}{c}0.035(4) \\
{[63 \%]}\end{array}$ & $\begin{array}{c}0.007(2) \\
{[13 \%]}\end{array}$ & $\begin{array}{c}0.014(5) \\
{[25 \%]}\end{array}$ & $176.5(2)$ \\
\hline $\begin{array}{c}\mathrm{BaTiO}_{3}[37] \\
(300 \mathrm{~K})\end{array}$ & $-0.091(2)$ & $0.042(2)$ & $0.0985(16)$ & $\begin{array}{c}0.076(2) \\
{[62 \%]}\end{array}$ & $\begin{array}{c}-0.0087(17) \\
{[7 \%]}\end{array}$ & $\begin{array}{c}0.037(2) \\
{[30 \%]}\end{array}$ & $172.4(2)$ \\
\hline $\begin{array}{c}\mathrm{KNbO}_{3}[19] \\
(543 \mathrm{~K})\end{array}$ & $-0.09(4)$ & $0.08(4)$ & $0.07(4)$ & $\begin{array}{c}0.11(5) \\
{[86 \%]}\end{array}$ & $\begin{array}{c}-0.008(19) \\
{[6 \%]}\end{array}$ & $\begin{array}{c}-0.01(5) \\
{[8 \%]}\end{array}$ & $170.2(3)$ \\
\hline $\begin{array}{c}\mathrm{PbTiO}_{3}[38] \\
(295 \mathrm{~K})\end{array}$ & $0.1567(16)$ & $0.4879(13)$ & $0.4646(13)$ & $\begin{array}{c}0.1615(15) \\
{[41 \%]}\end{array}$ & $\begin{array}{c}0.2178(12) \\
{[55 \%]}\end{array}$ & $\begin{array}{c}-0.0155(15) \\
{[4 \%]}\end{array}$ & $160.7(2)$ \\
\hline $\begin{array}{c}\mathrm{BiCoO}_{3}[39] \\
(300 \mathrm{~K})\end{array}$ & $0.316(4)$ & $1.086(2)$ & $0.961(2)$ & $\begin{array}{c}0.402(4) \\
{[44 \%]}\end{array}$ & $\begin{array}{c}0.425(3) \\
{[47 \%]}\end{array}$ & $\begin{array}{c}-0.084(3) \\
{[9 \%]}\end{array}$ & $135.1(2)$ \\
\hline
\end{tabular}

with other ferroelectric perovskite materials. At the structural phase transition from cubic $P m 3 m$ to tetragonal $P 4 m m$ in the perovskite oxide, the polar vibrational motion is decomposed by three modes, so-called Slater, Last, and Axe modes [see Fig. 3(c)]. The analysis of these ferroelectric modes is commonly performed to classify the soft phonon mode obtained with the optical, x-ray, and neutron spectroscopy experiments [40-45]. In this paper, we use polar atomic displacements from centrosymmetric positions to estimate frozen ferroelectric modes. The frozen ferroelectric modes can be quantified from the masses and the atomic displacements from the centrosymmetric positions of ions, as Harada et al. did using the inelastic structure factor of the soft phonon modes [46]. Here, the polar atomic displacements of the $B, \mathrm{O} 1$, and $\mathrm{O} 2$ sites are represented by $\xi_{B}, \xi_{\mathrm{O} 1}$, and $\xi_{\mathrm{O} 2}$, respectively. The coefficients of the ferroelectric modes, $S_{\text {Slater }}, S_{\text {Last }}$, and $S_{\text {Axe }}$, can be defined as

$$
\begin{aligned}
& \xi_{x}=S_{\text {Slater }} \cdot s_{\text {Slater }}+S_{\text {Last }} \cdot s_{\text {Last }}+S_{\text {Axe }} \cdot s_{\text {Axe }}, \\
& \xi_{x}=\left(\xi_{B}, \xi_{\mathrm{O} 1}, \xi_{\mathrm{O} 2}\right) .
\end{aligned}
$$

Here, $\quad s_{\text {Slater }}=(-k, 1,1), \quad s_{\text {Last }}=\left(1+k^{\prime}, 1+k^{\prime}, 1+k^{\prime}\right)$, $s_{\text {Axe }}=(0,-1 / 2,1), \quad k=3 M_{\mathrm{O}} / M_{B}, \quad$ and $\quad k^{\prime}=\left(M_{B}+\right.$ $\left.3 M_{\mathrm{O}}\right) / M_{A} . M_{A}, M_{B}$, and $M_{\mathrm{O}}$ stand for the masses of the $A$-site, $B$-site, and $\mathrm{O}$ ions, respectively. Thus,

$$
\begin{aligned}
\left(\begin{array}{l}
\xi_{B} \\
\xi_{\mathrm{O} 1} \\
\xi_{\mathrm{O} 2}
\end{array}\right) & =\left(\begin{array}{ccc}
-k & 1+k^{\prime} & 0 \\
1 & 1+k^{\prime} & -\frac{1}{2} \\
1 & 1+k^{\prime} & 1
\end{array}\right)\left(\begin{array}{c}
S_{\text {Slater }} \\
S_{\text {Last }} \\
S_{\text {Axe }}
\end{array}\right), \\
\left(\begin{array}{c}
S_{\text {Slater }} \\
S_{\text {Last }} \\
S_{\text {Axe }}
\end{array}\right) & =\alpha\left(\begin{array}{c}
\xi_{B} \\
\xi_{\mathrm{O} 1} \\
\xi_{\mathrm{O} 2}
\end{array}\right), \\
\alpha & =\frac{1}{3(1+k)}\left(\begin{array}{ccc}
-3 & 2 & 1 \\
\frac{3}{1+k^{\prime}} & \frac{2 k}{1+k^{\prime}} & \frac{k}{1+k^{\prime}} \\
0 & -2(1+k) & 2(1+k)
\end{array}\right) .
\end{aligned}
$$

The magnitude of the ferroelectric polarization based on the point-charge model for each mode has a proportional relation to the coefficient for the corresponding mode in Eq. (3). In Table III, the coefficients of the ferroelectric modes and their ratio are summarized for the tetragonal perovskite $\mathrm{Sr}_{1 / 2} \mathrm{Ba}_{1 / 2} \mathrm{MnO}_{3}$ in comparison with other perovskite ferroelectrics, tetragonal $\mathrm{BaTiO}_{3}, \mathrm{KNbO}_{3}, \mathrm{PbTiO}_{3}$, and $\mathrm{BiCoO}_{3}$ [19,37-39].

In the ferroelectric phase of the tetragonal perovskite $\mathrm{Sr}_{1 / 2} \mathrm{Ba}_{1 / 2} \mathrm{MnO}_{3}$, the dominant positive $S_{\text {Slater }}$, relatively large negative $S_{\text {Last }}$, and small positive $S_{\text {Axe }}$ are obtained. The contributions from $\left|S_{\text {Slater }}\right|,\left|S_{\text {Last }}\right|$, and $\left|S_{\text {Axe }}\right|$ are approximately $70 \%, 18 \%$, and $12 \%$, compatible with the result $(71 \%, 24 \%$, and $5 \%$ ) obtained with the optical and inelastic $\mathrm{x}$-ray spectroscopies [45]. In tetragonal $\mathrm{BaTiO}_{3}$, the dominant positive $S_{\text {Slater }}$, small negative $S_{\text {Last }}$, and relatively large positive $S_{\text {Axe }}$ are observed. In tetragonal $\mathrm{KNbO}_{3}$, the positive $S_{\text {Slater }}$ is dominant, but the error bars for the other modes are too large. As a commonality, they share two characteristics, the dominant positive $S_{\text {Slater }}$ and negative $S_{\text {Last }}$.

To explain the origin of the commonality, we refer to earlier first-principles calculations for perovskite oxides, which pointed out the importance of the covalency between the $B$-site and apical $\mathrm{O} 2$ ions for the emergence of ferroelectricity $[1,47-49]$. This is the reason why the dominant parameter is the Slater mode when contracting the distance between the $B$-site and $\mathrm{O} 2$ ions, as shown in Fig. 3(c). The negative $S_{\text {Last }}$ and positive $S_{\text {Axe }}$ play a role in reducing the extra atomic displacements of the $\mathrm{O} 1$ ions generated by the Slater mode.

In $\mathrm{PbTiO}_{3}$, the earlier first-principles calculation also pointed out that the hybridization between the $6 p$ band of $\mathrm{Pb}$ and $2 p$ band of $\mathrm{O} 1$ induces an additional component of the ferroelectric polarization [48]. In that case, the distance between the $\mathrm{Pb}$ and $\mathrm{O} 1$ ions also decreases. This atomic displacement induces the combined $S_{\text {Slater }}$ and $S_{\text {Last }}$ mode, which can actually be seen in $\mathrm{PbTiO}_{3}$, as listed in Table III. In $\mathrm{BiCoO}_{3}$, since the ratio of the ferroelectric modes is similar to that of $\mathrm{PbTiO}_{3}$, we speculate that the origin of the ferroelectricity for $\mathrm{BiCoO}_{3}$ is the same as that for $\mathrm{PbTiO}_{3}$. 
In the multiferroic phase of $\mathrm{Sr}_{1 / 2} \mathrm{Ba}_{1 / 2} \mathrm{MnO}_{3}, S_{\text {Slater }}$ and $S_{\text {Last }}$ are suppressed, while $S_{\mathrm{Axe}}$ is enlarged. In addition, the sign of $S_{\text {Last }}$ changes to positive. The $G$-AFM exchange interaction prefers the $180^{\circ} \mathrm{O} 1-\mathrm{Mn}-\mathrm{O} 1$ bond angle, which is contradictory to the off-centering distortion of the O1-Mn-O1 bond angle. Therefore, the displacement of the $\mathrm{Mn}$ ion is suppressed and, consequently, gives rise to the decrease in the $S_{\text {Slater }}$ and $S_{\text {Last }}$ modes. In stark contrast, the apical O2 is relatively free from the restriction of the magnetic exchange interaction. Thus, we speculate that the atomic displacement for apical $\mathrm{O} 2$ is enlarged to obtain the gain of the covalency between $\mathrm{Mn}$ and $\mathrm{O} 2$, resulting in the enlarged $S_{\text {Axe }}$ parameter. To eliminate the extra atomic displacements of $\mathrm{O} 1$ from $S_{\mathrm{Axe}}$, the sign of the $S_{\text {Last }}$ mode changes to positive in the multiferroic phase. Using the experimentally determined crystalstructure information and the results of the mode analyses, we discussed and speculated on the qualitative suppression mechanism of the ferroelectricity. To support this speculation and provide more quantitative discussion, we performed a first-principles calculation.

\section{B. First-principles calculation}

For the discussion of the atomic displacements and the resulting ferroelectricity in this system, we performed first-principles calculations for the tetragonal perovskite $\mathrm{Sr}_{1 / 2} \mathrm{Ba}_{1 / 2} \mathrm{MnO}_{3}$. To understand the effect of the magnetic order on the ferroelectricity in the multiferroic phase, here, we also simulate the ferroelectric polarization in the hypothetical (energetically unfavored state determined from the first-principles calculation, see Appendix A) A-type antiferromagnetic (A-AFM) structure [the magnetic moments are aligned parallel in the $a-b$ plane with antiparallel coupling with neighboring planes, as shown in Fig. 1(b)] as well as the ground-state $G$-AFM structure in $\mathrm{Sr}_{1 / 2} \mathrm{Ba}_{1 / 2} \mathrm{MnO}_{3}$.

Figures 4(a) and 4(b) show the density of states from GGA $+U$ calculations. When we set $U=3 \mathrm{eV}$ and $J=1 \mathrm{eV}$, consistent with the previous DFT study [25], the system is an insulator while the energy gap is significantly underestimated as $E_{\text {gap }} \sim 0.5 \mathrm{eV}$, which is inconsistent with the experimentally estimated energy gap of $\sim 2 \mathrm{eV}$ for $\mathrm{SrMnO}_{3}$ [50]. The underestimation of the energy gap was not improved when the $U$ value was increased up to $6 \mathrm{eV}$ [see Fig. 4(b)]; on the contrary, the band gap was reduced to $\sim 0.3 \mathrm{eV}$. This result might seem counterintuitive, but it is due to a property of the GGA $+U$ method that adds the effective Coulomb potential only to the localized orbital states (such as the $3 d$ and $4 f$ orbital states). Indeed, the GGA $+U$ Coulomb potential shifts down the occupied Mn $3 d$ states but keeps delocalized O $2 p$ states at the original energy levels around the valence top state (note that the on-site Hartree-Fock-like interaction affects only the localized orbital states and does not affect the itinerant states) [34]. When the $\mathrm{O} 2 p$ states are located at the shallow energy level, the Mn ion displays a tendency to be in the trivalent instead of quadrivalent ionic state in the simulation. This is the reason the band gap tends to be closed with increasing the $U$ value. To make matters worse, this narrow energy gap is closed when the hypothetical ferromagnetic phase or A-AFM phase is calculated. Therefore, we conclude that the GGA + $U$ approach is not appropriate to describe the wide-gapped

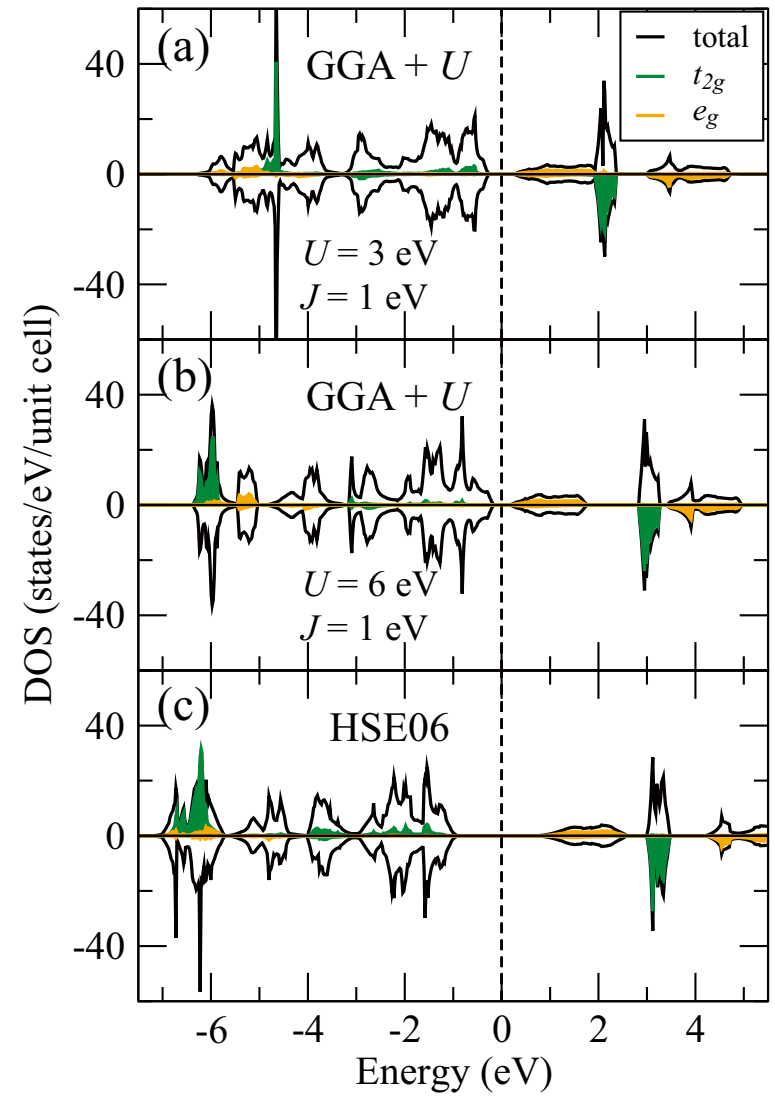

FIG. 4. Density of states (DOS) in G-AFM of $\mathrm{Sr}_{1 / 2} \mathrm{Ba}_{1 / 2} \mathrm{MnO}_{3}$ calculated from the GGA $+U$ method with (a) $U=3 \mathrm{eV}$ and $J$ $=1 \mathrm{eV}$ and (b) $U=6 \mathrm{eV}$ and $J=1 \mathrm{eV}$ and from (c) the HSE06 method. Top and bottom panels show majority- and minority-spin states, respectively. Projected DOSs for $\mathrm{Mn} t_{2 g}$ and $e_{g}$ orbital states are highlighted in green and orange, respectively.

insulating state and evaluate the ferroelectric distortion in $\mathrm{Sr}_{1 / 2} \mathrm{Ba}_{1 / 2} \mathrm{MnO}_{3}$.

Figure 4(c) shows the density of states from the HSE06 calculation, leading to the wider energy gap $\left(E_{\text {gap }} \sim 2 \mathrm{eV}\right.$, consistent with the experimental data in $\mathrm{SrMnO}_{3}$ [50]) with the Mn quadrivalent state. In this case, the fraction of exact Hartree-Fock exchange in the HSE06 scheme shifts down both the occupied Mn $3 d$ levels and O $2 p$ levels. Hereinafter, we will focus on HSE06 results and discuss the ferroelectric property. By using the experimental and DFT-optimized crystal structures, the ferroelectric polarization was calculated as listed in Table IV. In order to investigate the influence of the magnetic ordering on the ferroelectric polarization, we consider the ground-state $G$-AFM and the hypothetical $A$-AFM configurations.

It is noted that the calculated ferroelectric polarization of the optimized structure based on the point-charge model with nominal ionic charges ( $\mathrm{Ba}$ and $\mathrm{Sr}, 2+; \mathrm{Mn}, 4+; \mathrm{O}, 2-$ ), i.e., the ionic displacement contribution to the ferroelectric polarization, shows good agreement with that estimated by the experimental crystal structure: $P_{\mathrm{PCM}} \sim 10.1 \mu \mathrm{C} / \mathrm{cm}^{2}$ with $G$-AFM in both the experimental and optimized structures at $T=50 \mathrm{~K}$. This result supports the advantage of using the HSE06 functional for the polar structural distortion with a 
TABLE IV. Calculated ferroelectric polarization for the experimental (E) and optimized (O) crystal structure at $T=50 \mathrm{~K}$ for the $G$-AFM and $A$-AFM antiferromagnetic ordering based on the HSE06 exchange-correlation functional. Both the net ferroelectric polarization obtained by the Berry phase method $P_{\text {Berry }}$ and the ionic contribution based on the point-charge model $P_{\mathrm{PCM}}$ are shown (in units of $\left.\mu \mathrm{C} / \mathrm{cm}^{2}\right)$.

\begin{tabular}{lcccc}
\hline \hline & $\mathrm{E}_{\mathrm{G}-\mathrm{AFM}}$ & $\mathrm{E}_{\mathrm{A}-\mathrm{AFM}}$ & $\mathrm{O}_{\mathrm{G}-\mathrm{AFM}}$ & $\mathrm{O}_{\mathrm{A}-\mathrm{AFM}}$ \\
\hline$P_{\text {Berry }}$ & 19.37 & 23.65 & 20.17 & 30.24 \\
$P_{\mathrm{PCM}}$ & 10.05 & 10.05 & 10.11 & 15.45 \\
\hline \hline
\end{tabular}

high accuracy. The total ferroelectric polarization $P_{\text {Berry }}$, i.e., the summation of ionic and electronic contributions, is almost double $P_{\mathrm{PCM}}$, as is often seen in other ferroelectric manganites [51], and is of the same order of magnitude as the ferroelectric polarization $\left(13.5 \mu \mathrm{C} / \mathrm{cm}^{2}\right)$ experimentally obtained in the earlier study [18].

Next, we focus on the suppression mechanism of the ferroelectricity in the multiferroic phase. Basically, we consider two mechanisms to induce the ferroelectric polarization: hybridization between Mn $3 d$ and apical O2 $2 p$ states $\left(P_{\text {hyb }}\right)$ and in-plane Mn-O1-Mn magnetic exchange striction $\left(P_{\text {extr }}\right)$, as shown in Fig. 5. The $p$ - $d$ hybridization drives the polar ionic distortion of the Slater mode by which $\mathrm{Mn}$ and $\mathrm{O} 2$ ions are, respectively, shifted downward and upward. The magnetic exchange striction modulates the in-plane Mn-O1-Mn bond angle $\phi$, resulting in the suppression of $S_{\text {Slater }}$ and the sign
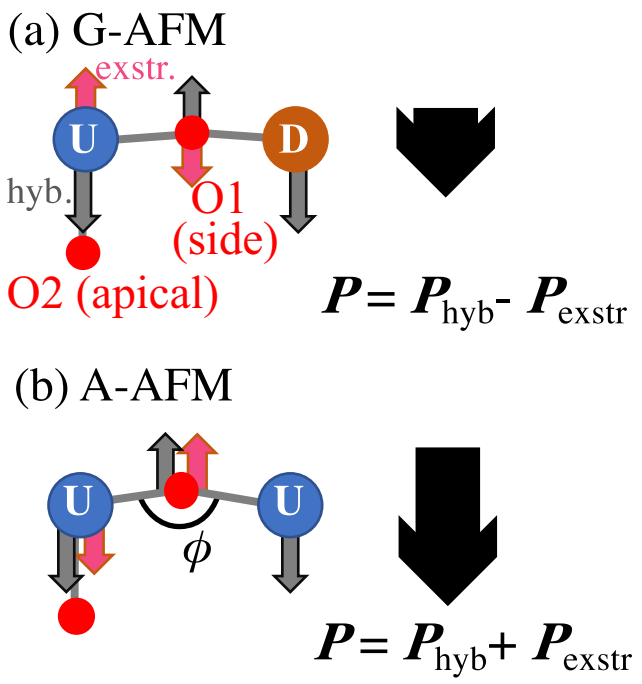

FIG. 5. Schematic illustration of ionic distortion (narrow gray and pink arrows) and induced ferroelectric polarization $P$ (wide black arrows) for (a) $G$-type $(G$-AFM) and (b) $A$-type ( $A$-AFM) antiferromagnetic orderings. Narrow gray and pink arrows stand for the atomic displacement originating from the hybridization between Mn $3 d$ and apical O2 $2 p$ bands and the in-plane Mn-O1-Mn magnetic exchange striction, respectively. $P_{\text {hyb }}$ and $P_{\text {exstr }}$ stand for the ferroelectric polarization from the hybridization and the exchange striction, respectively. U and D denote up- and down-spin Mn sites, respectively. Detailed crystal and magnetic structures are shown in Fig. 1(b). change of $S_{\text {Last }}$. Since the driving mechanism of the change in the ferroelectricity upon the ferroelectric to multiferroic phase transition is the magnetic exchange interaction, the atomic displacement should depend on the Mn spin configuration. In $G$-AFM, the magnetic exchange striction favors $\phi=180^{\circ}$, so Goodenough-Kanamori rule is satisfied for the $\mathrm{Mn}^{4+}$ ion [52-54]. This magnetic exchange striction prevents the atomic displacement of the side $\mathrm{O} 1$ ion, so that total ferroelectric polarization is reduced. In contrast, in the case of the hypothetical $A$-AFM, the magnetic exchange striction favors $\phi=90^{\circ}$ and enhances the hybridization-induced ferroelectric polarization, as shown in Fig. 5. The calculated ferroelectric polarization with $G$-AFM and $A$-AFM is $P_{\text {Berry }}=$ 20.17 and $30.24 \mu \mathrm{C} / \mathrm{cm}^{2}$, respectively, consistent with the above-discussed mechanism. The difference in the $P$ values allows us to decompose the ferroelectric polarization into two contributions, $P_{\text {hyb }} \sim 25$ and $P_{\text {extr }} \sim 5 \mu \mathrm{C} / \mathrm{cm}^{2}$. The former is comparable to the archetypal ferroelectric polarization in $\mathrm{BaTiO}_{3}\left(P \sim 26 \mu \mathrm{C} / \mathrm{cm}^{2}\right)$, and the latter is comparable to the magnetically driven ferroelectric polarization in multiferroic $\mathrm{HoMnO}_{3}\left(P \sim 6 \mu \mathrm{C} / \mathrm{cm}^{2}\right)$ [51]. Thus, we conclude that since only positive $P_{\text {hyb }}$ contributes to the ferroelectric polarization in the paramagnetic phase, negative $P_{\text {extr }}$ causes the suppression of the ferroelectric polarization observed in the multiferroic phase.

Last, we comment on the total energy difference between the ground-state G-AFM and hypothetical-state $A$-AFM. If one succeeded in stabilizing $A$-AFM in the tetragonal $A \mathrm{MnO}_{3}$ system, it might be a milestone multiferroic demonstrating ferroelectric polarization larger than the representative ferroelectric $\mathrm{BaTiO}_{3}$. Nonetheless, since $A$-AFM is energetically unfavored by $\sim 40 \mathrm{meV} /$ f.u. with respect to $G$-AFM, a study to stabilize the $A$-AFM state in $\mathrm{BaMnO}_{3}$ is left as a subject for future work. For the detailed calculation results and a comparison with other magnetic ordered states, see Appendix A.

\section{SUMMARY}

In summary, we have performed a synchrotron x-ray diffraction experiment to investigate accurate crystal structures in the ferroelectric and multiferroic phases of the tetragonal perovskite $\mathrm{Sr}_{1 / 2} \mathrm{Ba}_{1 / 2} \mathrm{MnO}_{3}$ using a twin-free singlecrystalline sample. A large atomic displacement for the $\mathrm{Mn}$ ion was observed in the ferroelectric phase. In the multiferroic phase, by contrast, the atomic displacement for the $\mathrm{Mn}$ ion is suppressed, but those for $\mathrm{O}$ ions are enlarged. From the obtained crystal-structure parameters, ferroelectric mode analyses were carried out. In the ferroelectric phase, the atomic displacements can be decomposed as the dominant positive Slater, negative Last, and small positive Axe modes. The suppression of Slater and Last modes, the sign change of the Last mode, and the enlargement of the Axe mode were found in the multiferroic phase. The first-principles calculation using the HSE06 functional successfully described the wide-gap insulating electronic states and quantitatively reproduced the experimentally observed ferroelectric polarization. The calculated ferroelectric polarization was further decomposed into two parts relevant to the hybridization and exchange-striction mechanisms. 
TABLE V. Top: Total energy difference $(\mathrm{meV} / \mathrm{Mn})$ between several magnetic orders. The atomic structure was fixed as the one obtained by optimizing the structure under $G$-AFM order. Bottom: Intersite magnetic coupling constants $J_{i j}$ (nearest-neighboring couplings $J_{i j}^{1 \|}$ in the layer and $J_{i j}^{1 \perp}$ interlayer) and second-nearest coupling $J_{i j}^{2 \|}$ in the layer found by using the total energy difference obtained with the fixed atomic structure. The Néel temperature of $G$ AFM order $T_{\mathrm{N}}^{\mathrm{G}}(\mathrm{K})$ is obtained from the mean-field approximation: $k_{\mathrm{B}} T_{\mathrm{N}}^{\mathrm{G}} \sim 1 / 3\left(4 J_{i j}^{1 \|}+2 J_{i j}^{1 \perp}\right)$, where $k_{\mathrm{B}}$ is the Boltzmann constant.

\begin{tabular}{lcccc}
\hline \hline & FM & A-AFM & C-AFM & G-AFM \\
\hline GGA $+U$ & 0.0 & -28.5 & -60.7 & -85.1 \\
HSE06 & 0.0 & -27.5 & -47.8 & -65.1 \\
& $J_{i j}^{1 \|}$ & $J_{i j}^{1 \perp}$ & $J_{i j}^{2 \|}$ & $T_{\mathrm{N}}^{\mathrm{G}}$ \\
\hline GGA $+U$ & 14.67 & 13.24 & 0.25 & 329 \\
HSE06 & 10.68 & 11.17 & 0.64 & 252 \\
\hline \hline
\end{tabular}

\section{ACKNOWLEDGMENTS}

The authors are grateful to T. Arima and H. Katsumoto for fruitful discussions. The synchrotron x-ray diffraction experiment was performed at SPring-8 with approval of the JASRI (Proposals No. 2009B1304 and No. 2010A1795). This work was in part supported by Grant-in-Aids for Scientific Research (Grants No. 17H02916, No. 17K14327, No. 19K03709, and No. 19H05822) from the Ministry of Education, Culture, Sports, Science and Technology (MEXT), Japan, and by the Research Program for CORE laboratory of the Dynamic Alliance for Open Innovation Bridging Human, Environment and Materials in the Network Joint Research Center for Materials and Devices. The computation in this work has been partially done using the facilities of the $\mathrm{Su}$ percomputer Center, the Institute for Solid State Physics, the University of Tokyo.

\section{APPENDIX A: DFT RESULTS FOR MAGNETIC STABILITY}

In this Appendix and Appendix B, we respectively show the DFT results for magnetic stability and the convergency test with the result cutoff energy and number of $\boldsymbol{k}$ points for the tetragonal perovskite $\mathrm{Sr}_{1 / 2} \mathrm{Ba}_{1 / 2} \mathrm{MnO}_{3}$.

The magnetic stability in $\mathrm{Sr}_{1 / 2} \mathrm{Ba}_{1 / 2} \mathrm{MnO}_{3}$ is evaluated by comparing the total energies for ferromagnetic (FM) and antiferromagnetic (AFM) orders with $A$ - $(A$-AFM), $C$ - $(C$ AFM), and $G$-type ( $G$-AFM) configurations. The results are summarized in Table V. Magnetic exchange coupling constants $J_{i j}$ were obtained by mapping the calculated magnetic
TABLE VI. Calculated ferroelectric polarization for the optimized crystal structure at $T=50 \mathrm{~K}$ for the $G$-AFM order based on the HSE06 exchange-correlation functional. Both the net polarization obtained by the Berry phase method $P_{\text {Berry }}$ and the ionic contribution based on the point-charge model $P_{\mathrm{PCM}}$ are shown (in units of $\mu \mathrm{C} / \mathrm{cm}^{2}$ ). Those results with a cutoff energy of $500 \mathrm{eV}$ and with an $(8,8,6) \boldsymbol{k}$-point mesh are not shown here since the calculations did not converge within 14 days in real time.

\begin{tabular}{lcccc}
\hline \hline Cutoff energy (eV) & 200 & 300 & 400 & 500 \\
\hline$P_{\text {Berry }}$ & 0.00 & 19.48 & 20.17 & \\
$P_{\text {PCM }}$ & 0.00 & 9.62 & 10.11 & \\
$\boldsymbol{k}$-point mesh & $(2,2,2)$ & $(4,4,3)$ & $(6,6,4)$ & $(8,8,6)$ \\
\hline$P_{\text {Berry }}$ & 18.86 & 20.17 & 19.76 & \\
$P_{\text {PCM }}$ & 9.66 & 10.11 & 9.78 & \\
\hline
\end{tabular}

energy to the Heisenberg Hamiltonian, $\mathcal{H}=\sum_{\langle i, j\rangle} J_{i j} \boldsymbol{s}_{i} \cdot \boldsymbol{s}_{j}$, where $s_{i, j}$ is the unit vector parallel to Mn spin [28]. With both GGA $+U$ and HSE06 methods, the stablest magnetic order is $G$-AFM, consistent with the experimental observation in $\mathrm{SrMnO}_{3}$ [18]. The $G$-AFM order is stable owing to the strong interlayer and in-layer antiferromagnetic superexchange couplings, $J_{i j}^{1 \perp}$ and $J_{i j}^{1 \|}$, respectively. The antiferromagnetic superexchange behavior can be explained by GoodenoughKanamori rules in the case of the $\mathrm{Mn}^{4+}\left(3 d^{5}\right)-\mathrm{O}-\mathrm{Mn}^{4+}\left(3 d^{5}\right)$ straight bond [52,54]. Based on the mean-field approximation, the GGA $+U$ result shows a stable $G$-AFM order with a high Néel temperature $T_{\mathrm{N}}^{\mathrm{G}}=329 \mathrm{~K}$, overestimating the experimental value, $T_{\mathrm{N}}=185 \mathrm{~K}$. On the other hand, $T_{\mathrm{N}}^{\mathrm{G}}=$ $252 \mathrm{~K}$ calculated using the HSE06 method is more consistent with the experimental $T_{\mathrm{N}}$. The difference comes from the fact that the GGA $+U$ calculation tends to overestimate both the $p-d$ hybridization and the superexchange interactions. Such an overestimation is likely corrected in the HSE06 calculation.

\section{APPENDIX B: CONVERGENCY TEST WITH THE CUTOFF ENERGY AND NUMBER OF $\boldsymbol{k}$ POINTS}

The test calculations were performed to check the convergence of our simulations for cutoff energy and $\boldsymbol{k}$ points. Table VI shows the calculated ferroelectric polarization for the optimized crystal structure at $T=50 \mathrm{~K}$ for the $G$-AFM based on the HSE06 exchange-correlation functional as varying the cutoff energy and $\boldsymbol{k}$-point mesh. We found that it is enough to set cutoff energy as $400 \mathrm{eV}$ and the $\boldsymbol{k}$-point mesh as $(4,4,3)$ to reach the convergence of the calculated polarization.
[1] R. E. Cohen, Nature (London) 358, 136 (1992).

[2] R. D. King-Smith and D. Vanderbilt, Phys. Rev. B 49, 5828 (1994).

[3] W. Zhong, D. Vanderbilt, and K. M. Rabe, Phys. Rev. Lett. 73, 1861 (1994).

[4] P. Muralt, J. Micromech. Microeng. 10, 136 (2000).

[5] G. A. Samara, J. Phys.: Condens. Matter 15, R367 (2003).
[6] M. Dawber, K. M. Rabe, and J. F. Scott, Rev. Mod. Phys. 77, 1083 (2005).

[7] J. Harada, T. Pedersen, and Z. Barnea, Acta Crystallogr., Sect. A 26, 336 (1970).

[8] T. Kimura, T. Goto, H. Shintani, K. Ishizaka, T. Arima, and Y. Tokura, Nature (London) 426, 55 (2003).

[9] M. Fiebig, J. Phys. D 38, R123 (2005).

[10] S.-W. Cheong and M. Mostovoy, Nat. Mater. 6, 13 (2007). 
[11] Y. Tokura, S. Seki, and N. Nagaosa, Rep. Prog. Phys. 77, 076501 (2014).

[12] V. V. Shvartsman, W. Kleemann, R. Haumont, and J. Kreisel, Appl. Phys. Lett. 90, 172115 (2007).

[13] D. Lebeugle, D. Colson, A. Forget, and M. Viret, Appl. Phys. Lett. 91, 022907 (2007).

[14] J. H. Lee and R. S. Fishman, Phys. Rev. Lett. 115, 207203 (2015).

[15] S. Bhattacharjee, E. Bousquet, and P. Ghosez, Phys. Rev. Lett. 102, 117602 (2009).

[16] J. M. Rondinelli, A. S. Eidelson, and N. A. Spaldin, Phys. Rev. B 79, 205119 (2009).

[17] J. H. Lee and K. M. Rabe, Phys. Rev. Lett. 104, 207204 (2010).

[18] H. Sakai, J. Fujioka, T. Fukuda, D. Okuyama, D. Hashizume, F. Kagawa, H. Nakao, Y. Murakami, T. Arima, A. Q. R. Baron, Y. Taguchi, and Y. Tokura, Phys. Rev. Lett. 107, 137601 (2011).

[19] A. W. Hewat, J. Phys. C 6, 2559 (1973).

[20] B. C. Frazer, H. R. Danner, and R. Pepinsky, Phys. Rev. 100, 745 (1955).

[21] G. Shirane, H. Danner, and R. Pepinsky, Phys. Rev. 105, 856 (1957).

[22] K. Momma and F. Izumi, J. Appl. Crystallogr. 44, 1272 (2011).

[23] T. Takeda, and S. Ōhara, J. Phys. Soc. Jpn. 37, 275 (1974).

[24] O. Chmaissem, B. Dabrowski, S. Kolesnik, J. Mais, D. E. Brown, R. Kruk, P. Prior, B. Pyles, and J. D. Jorgensen, Phys. Rev. B 64, 134412 (2001).

[25] G. Giovannetti, S. Kumar, C. Ortix, M. Capone, and J. van den Brink, Phys. Rev. Lett. 109, 107601 (2012).

[26] D. Okuyama, S. Ishiwata, Y. Takahashi, K. Yamauchi, S. Picozzi, K. Sugimoto, H. Sakai, M. Takata, R. Shimano, Y. Taguchi, T. Arima, and Y. Tokura, Phys. Rev. B 84, 054440 (2011).

[27] K. Yamauchi, F. Freimuth, S. Blügel, and S. Picozzi, Phys. Rev. B 78, 014403 (2008).

[28] K. Yamauchi, T. Oguchi, and S. Picozzi, J. Phys. Soc. Jpn. 83, 094712 (2014).

[29] O. Prokhnenko, R. Feyerherm, E. Dudzik, S. Landsgesell, N. Aliouane, L. C. Chapon, and D. N. Argyriou, Phys. Rev. Lett. 98, 057206 (2007).

[30] S. Ishiwata, Y. Kaneko, Y. Tokunaga, Y. Taguchi, T.-H. Arima, and Y. Tokura, Phys. Rev. B 81, 100411(R) (2010).

[31] K. Sugimoto, H. Ohsumi, S. Aoyagi, E. Nishibori, C. Moriyoshi, Y. Kuroiwa, H. Sawa, and M. Takata, AIP Conf. Proc. 1234, 887 (2010).
[32] T. Higashi, ABSCOR: Empirical absorption correction based on Fourier series approximation, Rigaku Corporation, Tokyo, 1995.

[33] G. Kresse and J. Furthmüller, Phys. Rev. B 54, 11169 (1996).

[34] V. I. Anisimov, F. Aryasetiawan, and A. I. Lichtenstein, J. Phys.: Condens. Matter 9, 767 (1997).

[35] J. Heyd, G. E. Scuseria, and M. Ernzerhof, J. Chem. Phys. 118, 8207 (2003).

[36] A. Stroppa and S. Picozzi, Phys. Chem. Chem. Phys. 12, 5405 (2010).

[37] G. H. Kwei, A. C. Lawson, S. J. L. Billinge, and S.-W. Cheong, J. Phys. Chem. 97, 2368 (1993).

[38] R. J. Nelmes and W. F. Kuhs, Solid State Commun. 54, 721 (1985).

[39] A. A. Belik, S. Iikubo, K. Kodama, N. Igawa, S. Shamoto, S. Niitaka, M. Azuma, Y. Shimakawa, M. Takano, F. Izumi, and E. Takayama-Muromachi, Chem. Mater. 18, 798 (2006).

[40] W. Cochran, Adv. Phys. 9, 387 (1960).

[41] G. Shirane, J. D. Axe, J. Harada, and J. P. Remeika, Phys. Rev. B 2, 155 (1970).

[42] A. C. Nunes, J. D. Axe, and G. Shirane, Ferroelectrics 2, 291 (1971).

[43] J. Harada, J. D. Axe, and G. Shirane, Phys. Rev. B 4, 155 (1971).

[44] J. F. Scott, Rev. Mod. Phys. 46, 83 (1974).

[45] H. Sakai, J. Fujioka, T. Fukuda, M. S. Bahramy, D. Okuyama, R. Arita, T. Arima, A. Q. R. Baron, Y. Taguchi, and Y. Tokura, Phys. Rev. B 86, 104407 (2012).

[46] J. Harada, J. D. Axe, and G. Shirane, Acta Crystallogr., Sect. A 26, 608 (1970).

[47] R. E. Cohen and H. Krakauer, Ferroelectrics 136, 65 (1992).

[48] H. Miyazawa, E. Natori, S. Miyashita, T. Shimoda, F. Ishii, and T. Oguchi, Jpn. J. Appl. Phys. 39, 5679 (2000).

[49] A. Chassé, St. Borek, K.-M. Schindler, M. Trautmann, M. Huth, F. Steudel, L. Makhova, J. Gräfe, and R. Denecke, Phys. Rev. B 84, 195135 (2011).

[50] T. Saitoh, A. E. Bocquet, T. Mizokawa, H. Namatame, A. Fujimori, M. Abbate, Y. Takeda, and M. Takano, Phys. Rev. B 51, 13942 (1995).

[51] S. Picozzi, K. Yamauchi, B. Sanyal, I. A. Sergienko, and E. Dagotto, Phys. Rev. Lett. 99, 227201 (2007).

[52] J. B. Goodenough, Phys. Rev. 100, 564 (1955).

[53] J. B. Goodenough, J. Phys. Chem. Solids 6, 287 (1958).

[54] J. Kanamori, J. Phys. Chem. Solids 10, 87 (1959). 